\title{
Design and Simulation of Compact Band-Pass Filter Using Stub Loaded Plasmonic Mim Waveguide
}

\author{
M.Vishwanath ${ }^{1}$, Dr.Habibullakhan ${ }^{2}$ \\ ${ }^{1}$ Research scholar at Dept: of ECE, Konerulakshmaiah Education Foundation, Guntur \\ ${ }^{2}$ Professor at Dept: of ECE, Konerulakshmaiah Education Foundation, Guntur \\ *Corresponding author E-mail visuklu@gmail.com
}

\begin{abstract}
In this article, plasmonic band-pass filters (BPF) have been studied and numerically analyzed. This filter has been designed based on the two-stubs. Pass-band can be realized by appropriately adjusting the lengths and width of the resonator. Based on the ideal characteristics of the proposed two stubs BPF is allowing the band at THz frequencies. Multiple transmission zeros are generated to improve the selectivity of the filter. All simulated results have been studied using CST Microwave studio suite. Usually, the transmission effectiveness is revealed by the exact resonance condition, whichwill confirmalong with the numerical simulation or theoretical analysis. This article delivers a promising application for plasmonic BPFsin addition to plasmonic integrated circuits (PICs).
\end{abstract}

\section{Introduction}

Surface plasmonpolaritons (SPPs) would be the basic elements for propagating the electromagnetic waves which have been generated and enclosed at the surface of the metal-dielectric interface [1], which are additionallyconsideredto overcome the diffraction limit in the light at nanoscale domain [2]. Recently, nanoplasmonic metal-insulator-metal (MIM) waveguide is one of various research topics [3], which can be confirmed to be a most effectivetechnique for guiding the light with nanoscalemode confinement and comparatively low loss [4], [5]. Being well recognized, severalsubwavelength optical devices have been designed using nanoplasmonic MIM waveguide are generally studied and reported [6],[7], which can beobtained by simply coupling with different resonators. Various stub resonators are already studied and researched by theory and experiments, including $\mathrm{V}$ grooves [8], bends [9], diskresonators [10]. Still, most of these stub resonators are the symmetricalshape. Keeping this fact in mind, we first propose a nanoplasmonic stub loaded MIMSIR. In this article, adual bandpass-band filter has been pro posed and a nanoplasmonic MIMSIR with coupled stubs is investigated. The full-wave simulations are utilized to study the transmission functionality of the proposed device using CST Microwave studio suite. The transmission spectrum of BPFis realized with the lengths and width of the waveguide when the effectiveindex of the insulator is fixed. Additionally, the wavelengths and transmission spectrum of the dips and peak are discussed. The transmission and reflection coefficients of the nanoplasmonic MIM waveguide-based optical devices have been obtained at $\mathrm{THz}$ frequencieswith a controllable transmission spectrum, which contains potential application in photonic integrated circuits (PICs) [11].

\section{Analysis of the Dual band pass band filter}

\section{with coupled stubs}

Fig. 1shows the dual bandpass band filter with coupled stubs using plasmonic MIMSIR. The dual band resonator is formed by loading two similar open stubs at identical sides of plasmonic MIMSIR with the dimensions $W 1=80 \mathrm{~nm}, \mathrm{~W} 2=320 \mathrm{~nm}$, lengths $\mathrm{L} 1=1700 \mathrm{~nm}, \mathrm{~L} 2=150 \mathrm{~nm}, \mathrm{~L} 3=400 \mathrm{~nm}$.

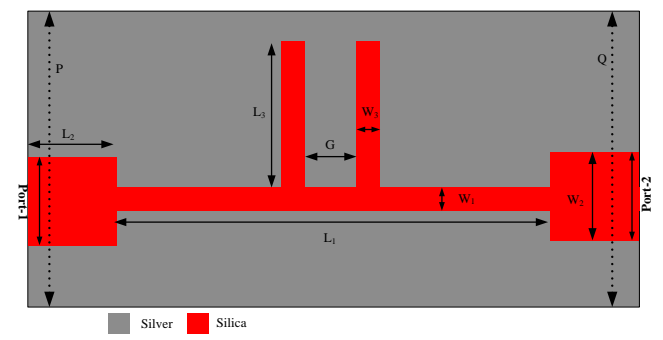

Fig.1 Geometry of the dual band Stub-Loaded MIMSIR based plasmonic Band Pass Filter for fixed widths $\mathrm{W}_{1}=80 \mathrm{~nm}, \mathrm{~W}_{2}=320 \mathrm{~nm}$, lengths $\mathrm{L}_{1}=1700 \mathrm{~nm}, \mathrm{~L}_{2}=150 \mathrm{~nm}$ $\mathrm{L}_{3}=400 \mathrm{~nm}$.

The high impedance gap is generally represented by widthW1and the lower impedance gap is represented by width W2, W3respectively,althoughMIMSIR waveguidetotal electrical length is $\mathrm{L} 1 * \theta 1$. P and Q are the power monitors, arranged for calculatingthe input and output power levels at same distances from the center point of the MTMSIR respectively.

From the simulationresults, the transverse magnetic (TM) mode with the nanoplasmonicwaveguide is usually excitedusing a dipole 
source and $5 \mathrm{~nm} \times 5 \mathrm{~nm}$ are the grid sizes along $\mathrm{x}$ and $\mathrm{y}$ directions. Here $\mathrm{W} 1>\mathrm{W} 3>\mathrm{W} 2$, as a result $\mathrm{Z} 1<\mathrm{Z} 3>\mathrm{Z} 2$ i.e. impedance is small , the width of the waveguide is largeandvice versa. Generally, for the admittance, resonance and spurious frequency of gap transmission line $(\lambda \mathrm{g} / 4)$ the suitable design equations for have been described in [12].

\section{Results and Discussion}

The filter response in addition to theresult of different modifications from width of MIM waveguide (W1) is shown in Fig.2 and Fig.3.

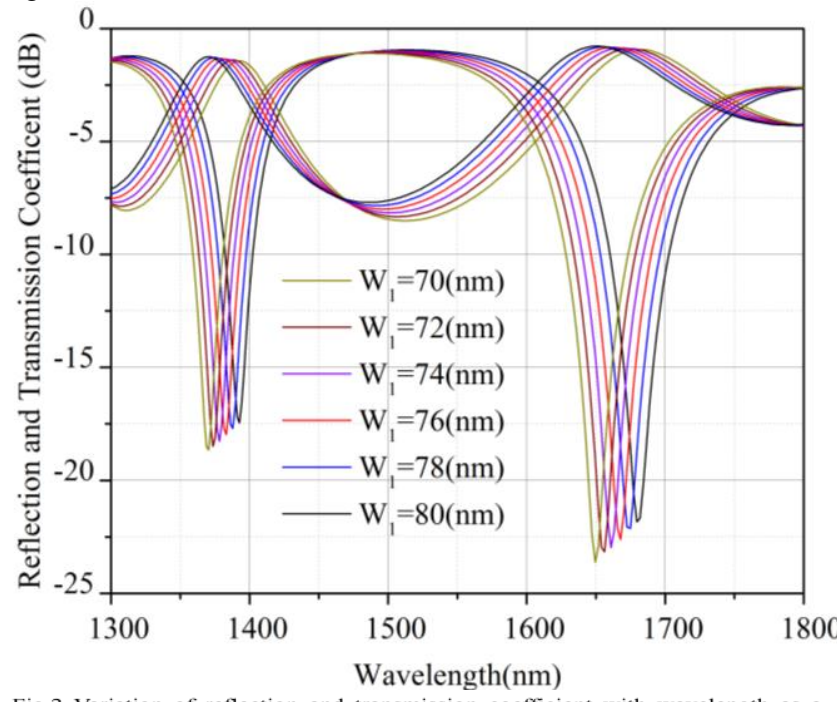

Fig. 2 Variation of reflection and transmission coefficient with wavelength as a
function of width(W), $W_{2}=320 \mathrm{~nm}$, lengths $L_{1}=1700 \mathrm{~nm}, L_{2}=150 \mathrm{~mm}, L_{3}=400$ nm.

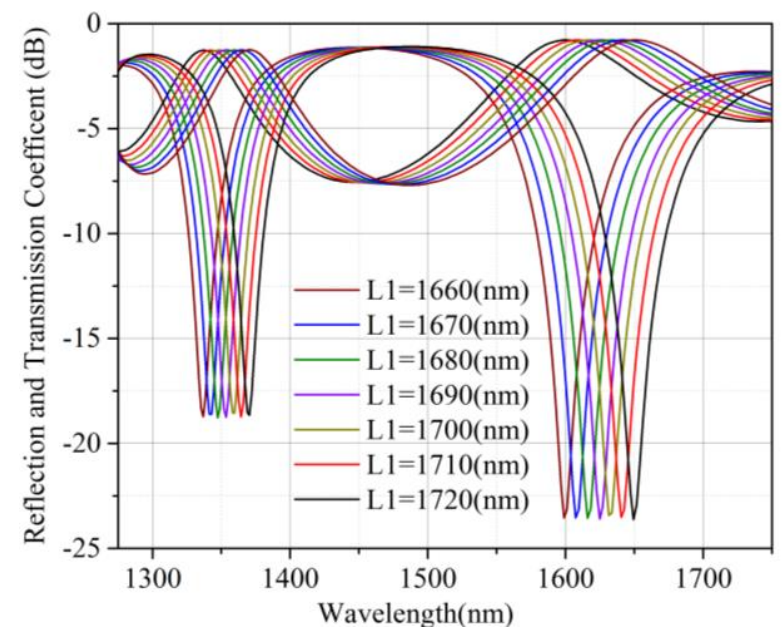

Fig.3 Variation of transmission and reflection coefficient with wavelength as a function of Length $\left(L_{1}\right), W_{1}=80 \mathrm{~nm}, W_{2}=320 \mathrm{~nm}$, lengths $L_{2}=150 \mathrm{~nm}, L_{3}=400 \mathrm{~nm}$.

A precise and unique passband is present around at 1358-nm and $1631-\mathrm{nm}$ in the proposed geometry; Fig. 4 depicts the field distributions of the recommended geometry.

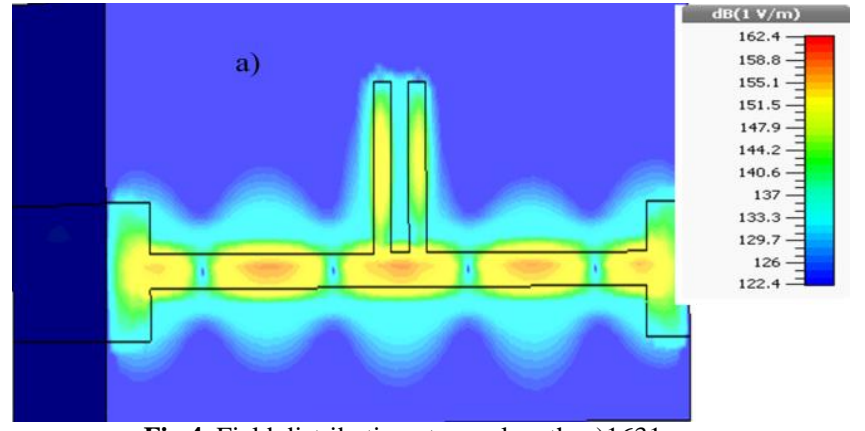

Fig.4 Field distribution at wavelength a)1631-nm

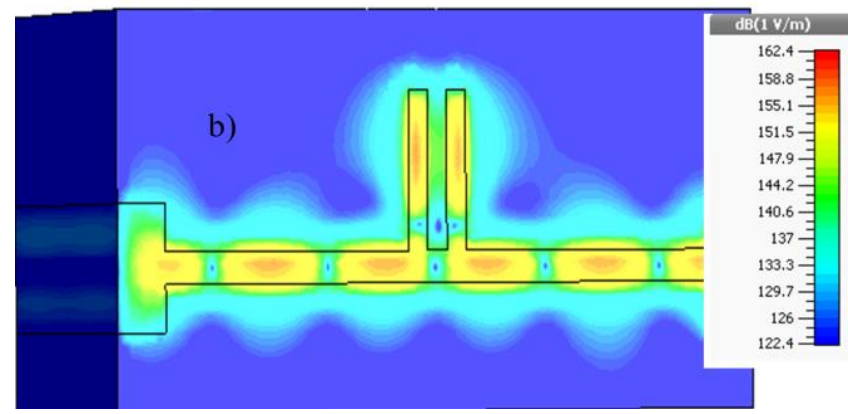

Fig.4 Field distribution at wavelength b)1358-nm

\section{Conclusions}

By utilizing a nanoplasmonic planar step impedance resonator in MIM gap waveguide, a newstub loaded plasmonic MIMSIR waveguide based dual band pass-band filter have been carried out and implemented. The simulation results of electromagnetic (EM) wave demonstratethe presence of two passbands at $1358 \mathrm{~nm}$ and $1631 \mathrm{~nm}$. The proposed pass-band filter device offers low insertion loss and is compact; thus it might be helpful in high-density multiband PICs.

\section{Acknowledgment}

The authors acknowledge support by the Koneru Lakshmaiah Educational Foundation, Guntur, Andhra Pradesh.

\section{References}

[1] [1] W. Mu and J. B. Ketterson, "Long-range surface plasmonpolaritons propagating on a dielectric waveguide support," Opt. Lett., vol. 36, no. 23, pp. 4713-4715, Dec. 2011.

[2] [2] W. Liu, R. Zhong, J. Zhou, Y. Zhang, and M. Hu, "Investigations on a nano-scale periodical waveguide structure taking surface plasmonpolaritons into consideration," J. Phys. D: Appl. Phys. vol. 45, no. 20, pp. 205104 (1-9), May 2012.

[3] [3] P. Taylor, C. Li, D. Qi, J. Xin, and F. Hao, "Metal-insulatormetal plasmonic waveguide for low- distortion slow light at telecom frequencies," J. Modern Opt. Vol. 61, no. 8, pp. 37-41, Nov.2014.

[4] [4] Jin Tao, Xu G. H, X Lin, Qin Zhang, and Xiaopin Jin, “A narrow-band subwavelengthplasmonic waveguide filter with asymmetrical multipleteeth-shaped structure," OPT. EXP.Vol. 17, No. 16, pp.13989-13994, Aug. 2009

[5] [5] B. H. Cheng, Y. Lai, and Y. Lan, "Plasmonic Photonic Bloch Oscillations in Composite Metal -Insulator -Metal Waveguide Structure," Plasmonics, vol. 9, no. 1, pp. 137-142, Jul. 2013.

[6] [6] G. Veronis, S. Fan, and S. Member, "Modes of SubwavelengthPlasmonic Slot Waveguides,” J. Lightwave Tech., vol. 25, no. 9, pp. 2511-2521, Sept. 2007.

[7] [7] A. Hosseini, A. Nieuwoudt, and Y. Massoud, "Efficient simulation of subwavelengthplasmonic waveguides using implicitly restarted Arnoldi," Opt. Express, vol. 14, no. 16, pp. 487-494, Aug. 2006

[8] [8] C. L. C. Smith, N. Stenger, A. Kristensen, and N. A. Mortensen, "Gap and channeled plasmons in tapered grooves : a review," The Royal Soc.Chem., vol. 7, no. 21, pp. 79-82, Apr. 2015. 
[9] [9] G. Veronis, S. Fan, G. Veronis, and S. Fan, "Bends and splitters in metal-dielectric-metal subwavelengthplasmonicwaveguides ," Appl. Phy. Lett. , vol. 87, , pp. 131102(1-4), Apr. 2005.

[10] [10] S. Randhawa et al., "Experimental demonstration of dielectricloaded plasmonic waveguide disk resonators at telecom wavelengths ," Appl. Phy. Lett. , vol. 98, ,pp. 161102 (1-3), Apr. 2011

[11] [11] X. Xiong et.al, "Broad band plasmonic absorber for photonic integrated circuits," IEEE Photn, Tech. Lett.Vol.26, No. 17, pp. 1726-1729, 2014

[12] [12] H. Lu, X. Liu, Y. Gong, L. Wang, and D. Mao, "Multi-channel plasmonic waveguide filters with disk-shaped nanocavities," Opt. Commun., vol. 284, no. 10-11, pp. 2613-2616, Feb. 2011. 